\title{
ALTERNATORS OF A RIGHT ALTERNATIVE ALGEBRA ${ }^{1}$ \\ BY \\ IRVIN ROY HENTZEL
}

\begin{abstract}
We show that in any right alternative algebra, the additive span of the alternators is nearly an ideal. We give an easy test to use to determine if a given set of additional identities will imply that the span of the alternators is an ideal. We apply our technique to the class of right alternative algebras satisfying the condition $(a, a, b)=\lambda[a,[a, b]]$. We show that any semiprime algebra over a field of characteristic $\neq 2, \neq 3$ which satisfies the right alternative law and the above identity with $\lambda \neq 0$ is a subdirect sum of (associative and commutative) integral domains.
\end{abstract}

Introduction. In any nonassociative algebra, the associator $(a, b, c)$ and the commutator $[a, b]$ are defined by: $(a, b, c)=(a b) c-a(b c) ;[a, b]=a b-$ $b a$. An alternator is an associator of the form $(a, a, b),(a, b, a)$, or $(b, a, a)$. We let $M$ represent the subspace generated by all alternators. If $R$ is a right alternative algebra, Thedy [5, Lemmas 11 and 12] has shown that $L=M+$ $R M$ is a left ideal and that $L$ contains a two-sided ideal of $R$. Furthermore, $M+M R$ is a two-sided ideal of $R$. There are known conditions which imply that $M$ is itself a left ideal; one such condition is the identity $[a,(b, b, a)] \subseteq$ $M[5$, p. 23]. We show exactly what identities are required to make $M$ a left ideal, and we give an easy way to deduce if a given right alternative algebra satisfies them. Some work [3] has been done on the structure of right alternative algebras where $M$ is assumed to be an ideal. Till now, the actual strength of this assumption was not known. We show that such an assumption is rather weak, and we give an easy process to see if $M$ is an ideal.

We finish by examining a variety of nonassociative algebras introduced by A. Sagle. These algebras arise from the Taylor series representation of an analytic multiplication on a manifold. They are right alternative algebras satisfying the additional identity

$$
(a, a, b)=\lambda[a,[a, b]] \text {. }
$$

When $\lambda=0$, equation (1) states that the algebra is alternative. If $\lambda \neq 0$ and the algebra is semiprime, then it must in fact be associative and commutative.

Presented to the Society, January 8, 1978; received by the editors January 18, 1977.

AMS (MOS) subject classifications (1970). Primary 17A30.

Key words and phrases. Right alternative, alternator ideal.

${ }^{1}$ This paper was partially supported by a grant from the Iowa State University Science and Humanities Research Institute. 
It is therefore a subdirect sum of integral domains. An integral domain is an associative-commutative ring satisfying $a b=0 \Rightarrow a=0$ or $b=0$.

We shall concern ourselves in this paper only with varieties of algebras. When we state that we find the conditions which imply that the span of the alternators of a right alternative algebra will be an ideal, we mean that we find the identities needed to define a variety of algebra which is a subvariety of right alternative algebras and in which the span of the alternators is an ideal.

Terminology. We draw heavily on the paper [2] for background showing how we use group representations to represent identities.

We also draw heavily on [5] for background results on right alternative rings.

Throughout the paper we will be studying algebras (not necessarily finite dimensional) over a field of characteristic different from 2 and 3. A right alternative algebra is a nonassociative algebra which is assumed to satisfy the identity

$$
0 \equiv \bar{A}(a, b, c)=(a, b, c)+(a, c, b)
$$

The following identities can be verified by expanding them. They hold in any nonassociative algebra.

$$
\begin{aligned}
0 \equiv & \bar{B}(a, b, c, d)=(a b, c, d)-(a, b c, d)+(a, b, c d) \\
& -a(b, c, d)-(a, b, c) d, \\
0 \equiv & \bar{C}(a, b, c)=[a b, c]-a[b, c]-[a, c] b-(a, b, c) \\
& +(a, c, b)-(c, a, b) .
\end{aligned}
$$

For convenience we define $\bar{M}(a, b, c)$ by

$$
\bar{M}(a, b, c)=(a, b, c)+(b, a, c) .
$$

In any right alternative ring

$$
\begin{gathered}
\bar{M}(a, b, c)=\bar{M}(b, a, c), \\
\bar{M}(a, b, c)+\bar{M}(b, c, a)+\bar{M}(c, a, b)=0 .
\end{gathered}
$$

We shall briefly review the technique of using representation theory of the symmetric group to study identities. We let $S_{n}$ be the group of permutations on $n$ distinct objects. Suppose $R$ is a nonassociative algebra over a field $F$. Let $f: \Pi_{i=1}^{n} R \rightarrow R$ be a function of $n$ arguments. For $\pi \in S_{n}$, by $f_{\pi}$ we mean to first permute the arguments by $\pi$ and then evaluate $f$ on them. For example, let $n=3$ and let $f$ be the associator $f(a, b, c)=(a b) c-a(b c)$. Then $f_{(12)}(a, b, c)=(b a) c-b(a c) ; f_{(123)}(a, b, c)=(c a) b-c(a b) ; f_{(12)}(a, b, a)=$ 
(ba) $a-b(a a)$. Let $G_{n}$ be the group ring on $S_{n}$ over $F$. If $g=\Sigma_{\pi \in S_{n}} \gamma_{\pi} \pi \in G_{n}$, then by $f_{g}$ we mean $\Sigma_{\pi \in S_{n}} \gamma_{\pi} f_{\pi}$.

If we have several functions $f_{j}$ : $\Pi_{i=1}^{n} R \rightarrow R, 1 \leqslant j \leqslant r$, then an element $g_{1} \oplus g_{2} \oplus \cdots \oplus g_{r}$ in the direct sum of $r$ copies of $G_{n}$ is called an identity of $R$ based on the functions $f_{1} \oplus f_{2} \oplus \cdots \oplus f_{r}$ if $\left(f_{1}\right)_{\left(g_{1}\right)}+\left(f_{2}\right)_{\left(g_{2}\right)}+\cdots+$ $\left(f_{r}\right)_{\left(g_{r}\right)}$ takes the value $0 \in R$ for all choices of its $n$ arguments. Let $L$ be the set of identities of $R$ based on the functions $f_{1} \oplus f_{2} \oplus \cdots \oplus f_{r}$. It is easy to show that $L$ forms a submodule over $G_{n}$. That is, if $g_{1} \oplus g_{2} \oplus \cdots \oplus g_{r}$ and $g_{1}^{\prime} \oplus g_{2}^{\prime} \oplus \cdots \oplus g_{r}^{\prime}$ are identities, then so is $g_{1}+g_{1}^{\prime} \oplus g_{2}+g_{2}^{\prime} \oplus \cdots \oplus g_{r}$ $+g_{r}^{\prime}$; if $g_{1} \oplus g_{2} \oplus \cdots \oplus g_{r}$ is an identity and $g \in G_{n}$, then $g g_{1} \oplus g g_{2}$ $\oplus \cdots \oplus g g_{r}$ is again an identity.

If the characteristic of the field $F$ is greater than $n$, then $G_{n}$ is isomorphic to a direct sum of complete matrix rings over $F$. The properties of the submodule of identities are expressible as properties of its summands.

If $\phi$ is a homomorphism of $G_{n}$ onto one of its direct summands, $K$, define $\phi: \prod_{i=1}^{r} G_{n} \rightarrow \prod_{i=1}^{r} K$ by

$$
\phi\left(g_{1} \oplus g_{2} \oplus \cdots \oplus g_{r}\right)=\phi\left(g_{1}\right) \oplus \phi\left(g_{2}\right) \oplus \cdots \oplus \phi\left(g_{r}\right) .
$$

$\phi(L)$ is a submodule over $\phi\left(G_{n}\right)=K$. Thus, if $k_{1} \oplus k_{2} \oplus \cdots \oplus k_{r}$ and $k_{1}^{\prime} \oplus k_{2}^{\prime} \oplus \cdots \oplus k_{r}^{\prime}$ are in $\phi(L)$, then so is $k_{1}+k_{1}^{\prime} \oplus k_{2}+k_{2}^{\prime} \oplus \cdots \oplus k_{r}$ $+k_{r}^{\prime}$; if $k \in K$ and $k_{1} \oplus k_{2} \oplus \cdots \oplus k_{r} \in \phi(L)$, then $k k_{1} \oplus k k_{2} \oplus \cdots \oplus$ $k k_{r} \in \phi(L)$.

Let $R S(\phi(L))$ be the collection of all rows which occur in some element of $\phi(L)$. The elements of $R S(\phi(L))$ are the full row of elements; they extend across $r$ matrices. $R S(\phi(L))$ is a vector space. Furthermore, $k_{1} \oplus k_{2}$ $\oplus \cdots \oplus k_{r}$ is in $\phi(L) \Leftrightarrow$ the rows of $k_{1} \oplus k_{2} \oplus \cdots \oplus k_{r}$ are in $R S(\phi(L))$. Since $R S(\phi(L))$ is a vector space, we can characterize it by giving a basis of its elements. In this paper, $L$ is generated by only a finite number of identities, say $l_{1}, l_{2}, \ldots, l_{j}$. Thus $R S(\phi(L))$ is spanned by the rows of $\phi\left(l_{1}\right)$, $\phi\left(l_{2}\right), \ldots, \phi\left(l_{j}\right)$. We derive a basis of $R S(\phi(L))$ by choosing a basis for the span of these rows. To choose this basis, we express the complete collection of rows of $\phi\left(l_{1}\right), \phi\left(l_{2}\right), \ldots, \phi\left(l_{j}\right)$ as a large matrix

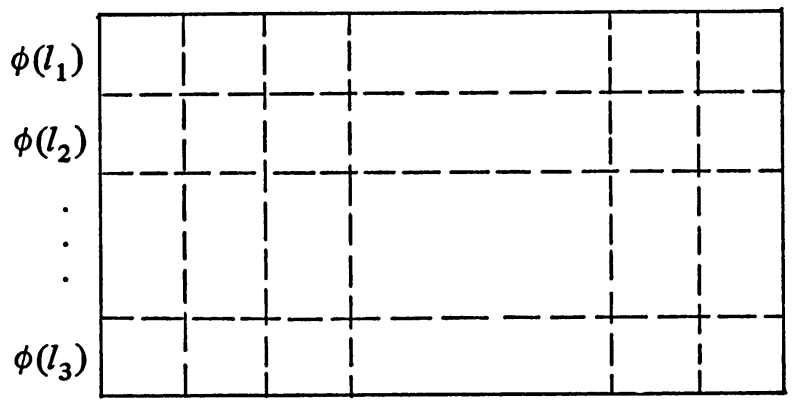


and reduce the large array to row canonical form.

Because $G_{n}$ is isomorphic to the direct sum of complete matrix rings, we know that $l$ is in $L \Leftrightarrow \phi(l)$ is in $\phi(L)$ for all summands. To prove an identity reduces to computing a basis for $\phi(L)$ for each summand. Then any proposed identity can be checked by seeing if, in each summand, its rows are linear combinations of the basis of $R S(\phi(L))$.

The listing of the basis of the identities of a right alternative ring is given in Table I. There are five representations called blocks, which are numbered 1 through 5. Each row of the table represents a minimal identity of degree 4. There are 25 of these minimal identities which characterize all degree 4 implications of the right alternative law. The functions used in Table $I$ are $\bar{M}(R R, R, R) \oplus(R, R, R R) \oplus R(R, R, R) \oplus[R,(R, R, R)]$.

We now describe how to convert a minimal identity from its representation as a row to an identity expressed in terms of the $r$ functions and various permutations of their arguments. Take any row, call it $R$, and suppose that $R$ comes from Block $x$. We will first construct an element $g_{1} \oplus g_{2} \oplus \cdots \oplus g_{r}$ whose row space in Block $x$ is spanned by $R$, and all other blocks are filled with zeros. Since there is a great deal of choice for the representation in the specified block, there are many ways to choose $g_{1} \oplus g_{2} \oplus \cdots \oplus g_{r}$. Of course, they will all be equivalent formulations of the same identity. The most direct way is to fill the top row in Block $x$ with $R$ and make all other entries zero. The identity $g_{1} \oplus g_{2} \oplus \cdots \oplus g_{r}$ generated for this choice might be more complicated than for another choice.

The calculation of $g_{1} \oplus g_{2} \oplus \cdots \oplus g_{r}$ is done using standard representation results [1, p. 24, Lemma 5.2].

THEOREM 1. Let $\left(a_{i j}\right)_{r x r}$ and $\left(b_{k l}\right)_{s x s}$ be two different summands of $G_{n}$. Then

(a) $\Sigma_{\pi \in S_{n}} b_{i j}\left(\pi^{-1}\right) a_{k l}(\pi)=0$,

(b) $\sum_{\pi \in S_{n}} a_{i j}\left(\pi^{-1}\right) a_{k l}(\pi)=\delta_{j k} \delta_{i l} n ! / r$,

where $\delta_{x y}$ is the Kronecker delta: $\delta_{x y}=0$ if $x \neq y, \delta_{x x}=1$.

We illustrate the technique using the first row of Block 3 of Table I:

$$
\begin{array}{lllllll}
1 & -1 & 0 & -3 & 0 & 0 & 0
\end{array}
$$

We wish to find $g_{1} \oplus g_{2} \oplus g_{3} \oplus g_{4}$ whose representation has all zeros in all blocks except in Block 3 , and whose representation in Block 3 is:

$$
\begin{array}{ccccccccccc}
1 & -1 & \oplus & 0 & -3 & 0 & 0 & 0 & \oplus & 0 & 1 \\
0 & 0 & 0 & 0
\end{array} 0^{0}
$$

Letting $\left(a_{i j}\right)$ be the representation of Block 3 , the $2 \times 2$ representation, then 


$$
\begin{aligned}
& g_{1}=(1 / 12) \sum_{\pi \in S_{4}}\left\{a_{11}\left(\pi^{-1}\right)-a_{21}\left(\pi^{-1}\right)\right\} \pi, \\
& g_{4}=(1 / 12) \sum_{\pi \in S_{4}} a_{21}\left(\pi^{-1}\right) \pi, \\
& g_{3}=0 \\
& g_{2}=-3 g_{4} .
\end{aligned}
$$

\section{TABLE I}

Identities of a right alternative algebra

$\bar{M}(R R, R, R) \quad \oplus \quad(R, R, R R) \oplus R(R, R, R) \oplus \quad[R,(R, R, R)]$

Block 1

Block 2

$$
-3
$$

\begin{tabular}{|c|c|c|c|c|c|}
\hline $\begin{array}{lll}1 & -1\end{array}$ & 0 & -3 & 0 & 0 & 0 \\
\hline $\begin{array}{ll}0 & 0\end{array}$ & 0 & 0 & 0 & 0 & 1 \\
\hline $\begin{array}{ll}0 & -1\end{array}$ & 0 & -2 & 0 & 1 & 0 \\
\hline 0 & 0 & 0 & 1 & 0 & 0 \\
\hline 0 & 1 & 0 & 0 & 0 & 0 \\
\hline
\end{tabular}

0

\begin{tabular}{|c|c|c|c|c|c|c|c|c|c|c|}
\hline$-3 / 4$ & $5 / 4$ & $1 / 4$ & 0 & & 0 & 0 & 0 & 0 & 0 & 0 \\
\hline $3 / 4$ & $-5 / 4$ & $-1 / 4$ & 0 & ) & 0 & 0 & 0 & 0 & 0 & 1 \\
\hline 0 & 0 & 0 & 0 & & 0 & 0 & 0 & 0 & 1 & 0 \\
\hline 0 & 1 & 0 & 0 & ) & 0 & 0 & 0 & 1 & 0 & 0 \\
\hline 0 & -1 & 0 & 0 & & 0 & 0 & 1 & 0 & 0 & 0 \\
\hline 0 & 0 & 0 & 0 & & 0 & 1 & 0 & 0 & 0 & 0 \\
\hline $3 / 4$ & $-1 / 4$ & $-1 / 4$ & 0 & & 1 & 0 & 0 & 0 & 0 & 0 \\
\hline$-1 / 4$ & $-1 / 4$ & $-1 / 4$ & 0 & & 0 & 0 & 0 & 0 & 0 & 0 \\
\hline$-1 / 2$ & $1 / 2$ & $1 / 2$ & 1 & ) & 0 & 0 & 0 & 0 & 0 & 0 \\
\hline
\end{tabular}

1

Block 3

\begin{tabular}{|c|c|c|c|c|c|c|c|c|}
\hline 1 & -2 & $0-1$ & 0 & -1 & 0 & 0 & 0 & 0 \\
\hline 1 & -2 & $0-1$ & 0 & -1 & 0 & 0 & 0 & 1 \\
\hline 2 & -2 & $0-1$ & 1 & -2 & 0 & 0 & 1 & 0 \\
\hline 0 & -1 & $0 \quad 0$ & 0 & -1 & 0 & 1 & 0 & 0 \\
\hline 0 & -1 & $0 \quad 0$ & 0 & -1 & 1 & 0 & 0 & 0 \\
\hline-1 & 1 & 10 & 0 & 0 & 0 & 0 & 0 & 0 \\
\hline
\end{tabular}

Block 4

Block 5 
We compute $g_{1}$ and $g_{4}$ using Table III in [2].

$$
\begin{gathered}
g_{1}=1 / 12\{2(12)-(13)-(14)-(23)-(24)+2(34)-(1432)-(1342) \\
+2(1423)-(1243)+2(1324)-(1234)+I-2(132)+(142) \\
+(123)-2(143)-2(124)+(134)+(243)-2(234)+(12)(34) \\
+(13)(24)+(14)(23)\} . \\
g_{4}=1 / 12\{-(12)+(14)+(23)-(34)+(1342)-(1423)+(1243) \\
-(1324)+(132)-(142)-(123)+(143)+(124)-(134) \\
-(243)+(234)\} .
\end{gathered}
$$

The minimal identity corresponding to the row $1-10-300001$ in Block 3 is

$$
\bar{M}(a b, c, d)_{g_{1}}+(a, b, c d)_{g_{2}}+a(b, c, d)_{g_{3}}+[a,(b, c, d)]_{g_{4}}=0 .
$$

Expanded completely, the identity becomes:

$$
\begin{aligned}
1 / 12\{2 & \bar{M}(b a, c, d)-\bar{M}(c b, a, d)-\bar{M}(d b, c, a)-\bar{M}(a c, b, d) \\
- & \bar{M}(a d, c, b)+2 \bar{M}(a b, d, c)-\bar{M}(b c, d, a)-\bar{M}(b d, a, c) \\
+ & 2 \bar{M}(c d, b, a)-\bar{M}(c a, d, b)+2 \bar{M}(d c, a, b)-\bar{M}(d a, b, c) \\
+ & \bar{M}(a b, c, d)-2 \bar{M}(b c, a, d)+\bar{M}(b d, c, a)+\bar{M}(c a, b, d) \\
- & 2 \bar{M}(c b, d, a)-2 \bar{M}(d a, c, b)+\bar{M}(d b, a, c)+\bar{M}(a c, d, b) \\
& -2 \bar{M}(a d, b, c)+\bar{M}(b a, d, c)+\bar{M}(c d, a, b)+\bar{M}(d c, b, a)\} \\
-1 / 4\{- & (b, a, c d)+(d, b, c a)+(a, c, b d)-(a, b, d c)+(b, d, a c) \\
& -(c, d, b a)+(c, a, d b)-(d, c, a b)+(b, c, a d) \\
& -(b, d, c a)-(c, a, b d)+(c, b, d a)+(d, a, c b)-(d, b, a c) \\
& -(a, c, d b)+(a, d, b c)\} \\
+1 / 12\{- & {[b,(a, c, d)]+[d,(b, c, a)]+[a,(c, b, d)]-[a,(b, d, c)] } \\
+ & {[b,(d, a, c)]-[c,(d, b, a)]+[c,(a, d, b)]-[d,(c, a, b)] } \\
+ & {[b,(c, a, d)]-[b,(d, c, a)]-[c,(a, b, d)]+[c,(b, d, a)] } \\
+ & {[d,(a, c, b)]-[d,(b, a, c)]-[a,(c, d, b)]+[a,(d, b, c)]\} . }
\end{aligned}
$$

Because minimal identities are, in general, so complicated, we seldom 
compute them. They may be handled conveniently in their representation notation.

We base our study of right alternative algebras on the functions $\bar{M}(R R, R, R) \oplus(R, R, R R) \oplus R(R, R, R) \oplus[R,(R, R, R)]$. We encode the Teichmüller identity $\bar{B}(a, b, c, d)$; the right alternative law $a \bar{A}(b, c, d),[a$, $\bar{A}(b, c, d)] ;$ and $\bar{M}(a b, c, d)+\bar{M}(c, d, a b)+\bar{M}(d, a b, c)=0$. These generate all identities of degree four of a right alternative algebra. This was checked by rewriting all these identities with respect to the associated products $((R R) R) R \oplus(R(R R)) R . \oplus(R R)(R R) \oplus R(R(R R)) \oplus$ $R((R R) R)$ and showing that they generate the same submodule as the right alternative law itself.

Table I contains these identities. Table I is essentially Table VII in [2] except that a different basis was chosen. In Table I the identities have been reduced to row canonical form with the stair-step ones running from upper right to lower left. The asterisks appearing in Table I mean that the function is an identity itself in that representation. The entries in Block 1 can be ignored since no matter what they are, they map to $0 \in R$ by the functions. A complete listing of the representations is printed in [2, Table III]. We shall give the representations of the generators (12) and (1234) for the sake of completeness.

$(1) \oplus(-1) \oplus\left(\begin{array}{rr}1 & 0 \\ -1 & -1\end{array}\right) \oplus\left[\begin{array}{lll}-1 & 0 & 0 \\ -1 & 1 & 0 \\ -1 & 0 & 1\end{array}\right] \oplus\left[\begin{array}{rrr}1 & 0 & 0 \\ 1 & -1 & 0 \\ 1 & 0 & -1\end{array}\right]$,
$(1) \oplus(-1) \oplus\left(\begin{array}{rr}-1 & -1 \\ 0 & 1\end{array}\right) \oplus\left[\begin{array}{lll}-1 & 1 & 0 \\ -1 & 0 & 1 \\ -1 & 0 & 0\end{array}\right] \oplus\left[\begin{array}{rrr}1 & -1 & 0 \\ 1 & 0 & -1 \\ 1 & 0 & 0\end{array}\right]$.

Ideal properties of $M$. Let $R$ be a right alternative algebra; let $M$ be the subspace of $R$ generated by all alternators. $M$ will be a left ideal of $R$ if $R \bar{M}(R, R, R) \subseteq \bar{M}(R R, R, R)+\bar{M}(R, R R, R)+\bar{M}(R, R, R R)$. By equation (6) and equation (7), we can assume $R \bar{M}(R, R, R) \subseteq$ $\bar{M}(R R, R, R)$. The representation of $I+(23)$ is:

$$
I+(23) \quad(2) \oplus(0) \oplus\left(\begin{array}{ll}
1 & 1 \\
1 & 1
\end{array}\right) \oplus\left[\begin{array}{lll}
1 & 1 & 0 \\
1 & 1 & 0 \\
0 & 0 & 2
\end{array}\right] \oplus\left[\begin{array}{rrr}
1 & -1 & 0 \\
-1 & 1 & 0 \\
0 & 0 & 0
\end{array}\right]
$$

In Table II we list an identity constructed from Table I. This is done by filling the rows of each block with linear combinations of the rows from the corresponding blocks of Table I. The goal was to produce an identity of the form $g \oplus 0 \oplus I+(23) \oplus 0$. This result would show that $R \bar{M}(R, R, R) \subseteq$ $\bar{M}(R R, R, R)$. Except for Block 3, an element $g$ can be constructed. 


\begin{tabular}{lrrrrrr} 
& \multicolumn{3}{c}{ TABLE II } & & & \\
& $\bar{M}(R R, R, R)$ & $\oplus$ & $R(R, R, R)$ \\
Block 1 & \multicolumn{2}{c}{0} & \multicolumn{3}{c}{2} & \\
Block 2 & \multicolumn{2}{c}{0} & \multicolumn{2}{c}{0} \\
Block 3 & \multicolumn{2}{c}{$\alpha$} & $\beta$ & & 1 & 1 \\
& & $\alpha$ & $\beta$ & & 1 & 1 \\
& 0 & -1 & 0 & 1 & 1 & 0 \\
Block 4 & 0 & -1 & 0 & 1 & 1 & 0 \\
& 0 & 2 & 0 & 0 & 0 & 2 \\
& -1 & 0 & 1 & 1 & -1 & 0 \\
Block 5 & 1 & 0 & -1 & -1 & 1 & 0 \\
& 0 & 0 & 0 & 0 & 0 & 0
\end{tabular}

We can see that $M$ is a left ideal if and only if there is an identity of the form:

$$
\text { (Block 3) } \quad \alpha \beta \quad \begin{array}{llllll}
\alpha & 0 & 1 & 1 & 0 & 0 .
\end{array}
$$

In a right alternative algebra, from Table $I$, we know that no such identity holds. $M$ is not a left ideal in a right alternative algebra.

LEMMA 1. $M$ is not a left ideal in right alternative algebras. The assumption that $M$ be a left ideal requires the assumption of a minimal identity of degree four. The identity is of form equation (8).

To check if an additional identity (i.e., in addition to the identities from the right alternative law) implies that $M$ is a left ideal, express the identity with respect to the same functions as Table I. Compute the identities implied in Block 3. $M$ will be a left ideal if and only if an identity of the form equation (8) is in the span of these new identities together with those from the right alternative law.

It is, of course, possible to express the absorption formula as

$$
a \bar{M}(b, c, d)=\sum_{\pi \in S_{4}} \gamma_{\pi} \bar{M}(a b, c, d)_{\pi} .
$$

In most applications it is sufficient to know that $M$ is a left ideal. The formula will in general be so complicated that it is useless for further computations unless it is expressed in terms of the representations, which is what we already have.

To decide the conditions which make $M$ a two-sided ideal, we will assume that $M$ is a left ideal and that $[R, M] \subseteq M$. Assuming $M$ is a left ideal implies that an identity of form equation (8) holds in Block 3. We will then determine 
the additional identities required so that $I+(23)$ is an identity $\bmod M$ based on the function $[R,(R, R, R)]$.

From Table I we construct the identity listed in Table III.

TABLE III

\begin{tabular}{lcccccc} 
& $\bar{M}(R R, R, R)$ & $\oplus[R,(R, R, R)]$ \\
Block 1 & \multicolumn{2}{c}{0} & 2 & \\
Block 2 & & 0 & 0 & \\
& & & 0 & \\
Block 3 & $3 / 2 \alpha+1$ & $3 / 2 \beta+1 / 2$ & 1 & 1 \\
& $3 / 2 \alpha+1$ & $3 / 2 \beta+1 / 2$ & 1 & 1 \\
& $3 / 4$ & $-5 / 4$ & $-1 / 4$ & 1 & 1 & 0 \\
Block 4 & $3 / 4$ & $-5 / 4$ & $-1 / 4$ & 1 & 1 & 0 \\
& $-3 / 2$ & $5 / 2$ & $1 / 2$ & 0 & 0 & 2 \\
& $\delta$ & $\epsilon$ & $\eta$ & 1 & -1 & 0 \\
Block 5 & $-\delta$ & $-\epsilon$ & $-\eta$ & -1 & 1 & 0 \\
& 0 & 0 & 0 & 0 & 0 & 0
\end{tabular}

LEMMA 2. In a right alternative algebra, $M$ need not be a two-sided ideal. For $M$ to be a two-sided ideal requires the assumption of two additional identities which are minimal identities of degree four. They are of form equation (8) and equation (9).

PRoof. If $M$ is to be a two-sided ideal, it must be a left ideal which requires a minimal identity of degree four in Block 3. The assumption of such an identity will not be sufficient, since there is no identity of the form:

$$
\text { (Block 5) } \quad \delta \varepsilon \eta \quad 000000001-10 .
$$

For $M$ to be a two-sided ideal requires, in addition, the assumption of a second minimal identity; this one is in Block 5 . We can see that $M$ can be a left ideal without being a right ideal.

To determine if an identity implies that $M$ is an ideal, we simply check the representations in Block 3 and Block 5 to see if identities equation (8) and equation (9) are satisfied.

Thedy [5, Lemma 11] has shown that in a right alternative ring, $M+M R$ is an ideal. It follows that if $M$ is a right ideal, then $M$ is a two-sided ideal. For completeness, we give this direct proof that if $M$ is a right ideal, then $M$ is a left ideal.

If $M$ is a right ideal, then $\bar{M}(R, R, R) R \subseteq M$ which implies the assumption of an identity of the following type:

$$
\text { (Block 3) } \quad \begin{array}{llllllllll}
\mu & \nu & 0 & 0 & 1 & 1 & -1 & -1 .
\end{array}
$$


By Table I this identity implies

$$
\text { (Block 3) } \quad-2 \mu \quad-2 \quad-2 \nu \quad-1 \quad 00011100
$$

which is of type equation (8). If $M$ is a right ideal, then $M$ is a left ideal and is consequently a two-sided ideal.

COROLlARY 1 (THEDY). Let $R$ be a right alternative algebra.

(1) $M$ is a left ideal $\Leftrightarrow[a,(b, b, a)] \subseteq M$ for all $a, b \in R$.

(2) $M$ is a two-sided ideal $\Leftrightarrow[R, M] \subseteq M$.

Proof of (1). The assumption $[a,(b, b, a)] \subseteq M$ for all $a, b \in R$ is equivalent to the assumption of an identity of the form

$$
g \oplus\{I+(23)\}\{I+(14)\}
$$

based on the functions $\bar{M}(R R, R, R) \oplus[R,(R, R, R)]$. We will first show that if $M$ is a left ideal, then an element $g$ exists. From Table $I$ and equation (8) we construct the identity in Table IV.

TABLE IV

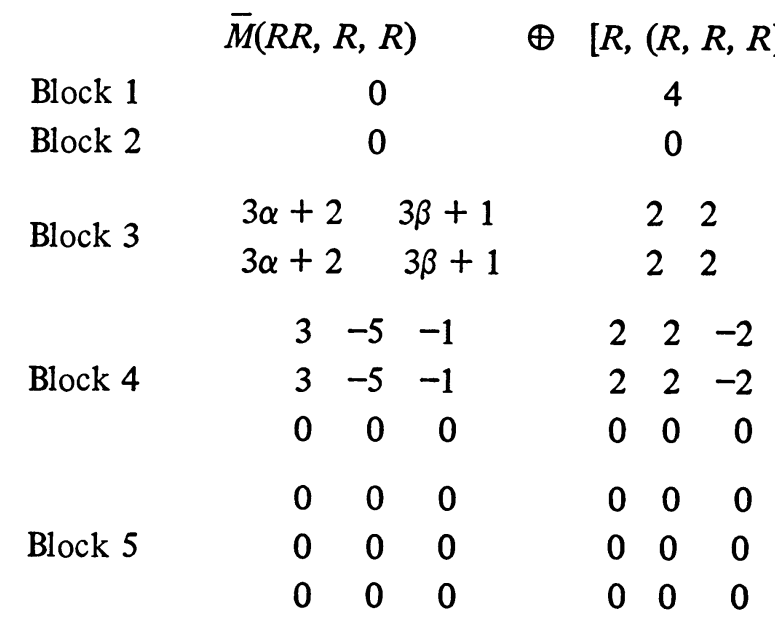

The entries under $[R,(R, R, R)]$ represent $\{I+(23)\}\{I+(14)\}$; the entries under $\bar{M}(R R, R, R)$ represent $g$. This shows that in any right alternative algebra, equation (8) implies (*). We shall now show that (*) implies an identity of form equation (8).

The equation (*) implies an identity of the following form.

(Block 3) $\quad \alpha^{\prime} \beta^{\prime} \quad 00000011$.

From Table I we see that this implies an identity of form equation (8). Note that we do not claim that every identity of form (*) is equivalent to equation (8) for some $\alpha, \beta$. We only claim that any such identity implies equation (8) for some $\alpha, \beta$, and that equation (8) implies some identity of form (*). 
Proof of (2). If $M$ is an ideal, then $[R, M] \subseteq M$ so the condition is necessary. If $[R, M] \subseteq M$, by part 1 of Corollary $1, M$ is a left ideal and hence a two-sided ideal.

Since the representation of $\{I+(14)\}\{I+(23)\}$ in Block 5 is zero, an identity $[a,(b, b, a)] \subseteq M$ for all $a, b$ in $R$ does not imply that $M$ is a two-sided ideal.

Corollary 1 is useful provided that the identities listed can be established. In general, it is often quicker to check for equations (8) and (9), since it is then immediate whether or not $M$ is a left or two-sided ideal or not.

Application. We now assume that for some fixed $\lambda$ in the field, the right alternative algebra satisfies an identity of form equation (1). We linearize equation (1) to obtain

$$
0 \equiv \bar{D}(a, b, c)=(a, b, c)+(b, a, c)-\lambda[a,[b, c]]-\lambda[b,[a, c]] .
$$

This identity is not expressible in terms of the functions $\bar{M}(R R, R, R) \oplus$ $(R, R, R R) \oplus R(R, R, R) \oplus[R,(R, R, R)]$ because it is not an identity implied by the associative law. We could express equation (11) and the right alternative law in terms of the functions $((R R) R) R \oplus(R(R R)) R \oplus$ $(R R)(R R) \oplus R(R(R R)) \oplus R((R R) R)$. We prefer, however, to keep our functions as some sort of associators as much as possible, even if this means studying a weaker system of identities.

LEMMA 3. If $R$ is a right alternative algebra satisfying equation (1), then $R$ satisfies the following identity:

$$
\begin{aligned}
0 \equiv & \bar{M}(a b, c, d)-a \bar{M}(b, c, d)-\bar{M}(a, c, d) b \\
& -\lambda([c, d], a, b)+2 \lambda([a, d], b, c) \\
& -2 \lambda(a, b,[c, d])-2 \lambda(a, c,[b, d]) \\
& +\lambda(c, a,[b, d])-\lambda(c, b,[a, d]) \\
& -2 \lambda[c,(a, b, d)]-\lambda[c,(d, a, b)]+\lambda[a, c][b, d]+\lambda[a, d][b, c] .
\end{aligned}
$$

Proof. $0 \equiv \bar{D}(a b, c, d)-a \bar{D}(b, c, d)-\bar{D}(a, c, d) b+\lambda \bar{C}(a, b,[c, d])+$ $\lambda[c, \bar{C}(a, b, d)]-\lambda \bar{C}(a,[b, d], c)-\lambda \bar{C}([\dot{a}, d], b, c)$.

This lengthy identity is easier seen in matrix representation. In Table $\mathrm{V}$ we display the permutations from which the matrices are calculated, as well as the matrix representations.

LEMMA 4. If $R$ is a right alternative algebra satisfying equation (1) for $\lambda \neq 0, \neq-\frac{1}{2}$, then $[a, b][c, d]=[c, d][a, b]$ for all $a, b, c, d$ in $R$.

Proof. We will show that the left ideal of identities based on the function $[R, R][R, R]$ contains $I-(13)(24)$. The matrix representation of $I-(13)(24)$ is 
$I-(13)(24) \quad(0) \oplus(0) \oplus\left(\begin{array}{ll}0 & 0 \\ 0 & 0\end{array}\right) \oplus\left[\begin{array}{rrr}1 & 1 & -1 \\ 0 & 2 & 0 \\ -1 & 1 & 1\end{array}\right] \oplus\left[\begin{array}{rrr}1 & 1 & -1 \\ 0 & 2 & 0 \\ -1 & 1 & 1\end{array}\right]$.

The left ideal of identities based on $[R, R][R, R]$ contains $I+(12)$ and $I+(34)$ and so contains

$$
\text { (1) } \oplus(0) \oplus\left(\begin{array}{ll}
1 & 0 \\
0 & 0
\end{array}\right) \oplus\left[\begin{array}{lll}
1 & 0 & 0 \\
0 & 1 & 0 \\
0 & 0 & 1
\end{array}\right] \oplus\left[\begin{array}{rrr}
1 & 0 & 0 \\
0 & 1 & -1 \\
0 & 0 & 0
\end{array}\right] \text {. }
$$

The proof will be complete if we demonstrate one more identity which holds in Block 5.

We refer to Table V. If we reduce the first row by the identities of Table I, and divide out the common factor $1+2 \lambda$, we get the identity:

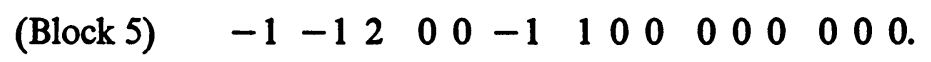

Reducing Row 3 of Block 5 using Table I gives

(Block 5)

$$
-1-\lambda-1-\lambda 2+2 \lambda \quad 00-1-\lambda \quad 1+\lambda \quad 0000000 \lambda 0-\lambda \text {. }
$$

\section{TABLE V}

Right alternative $\lambda$-algebra identities

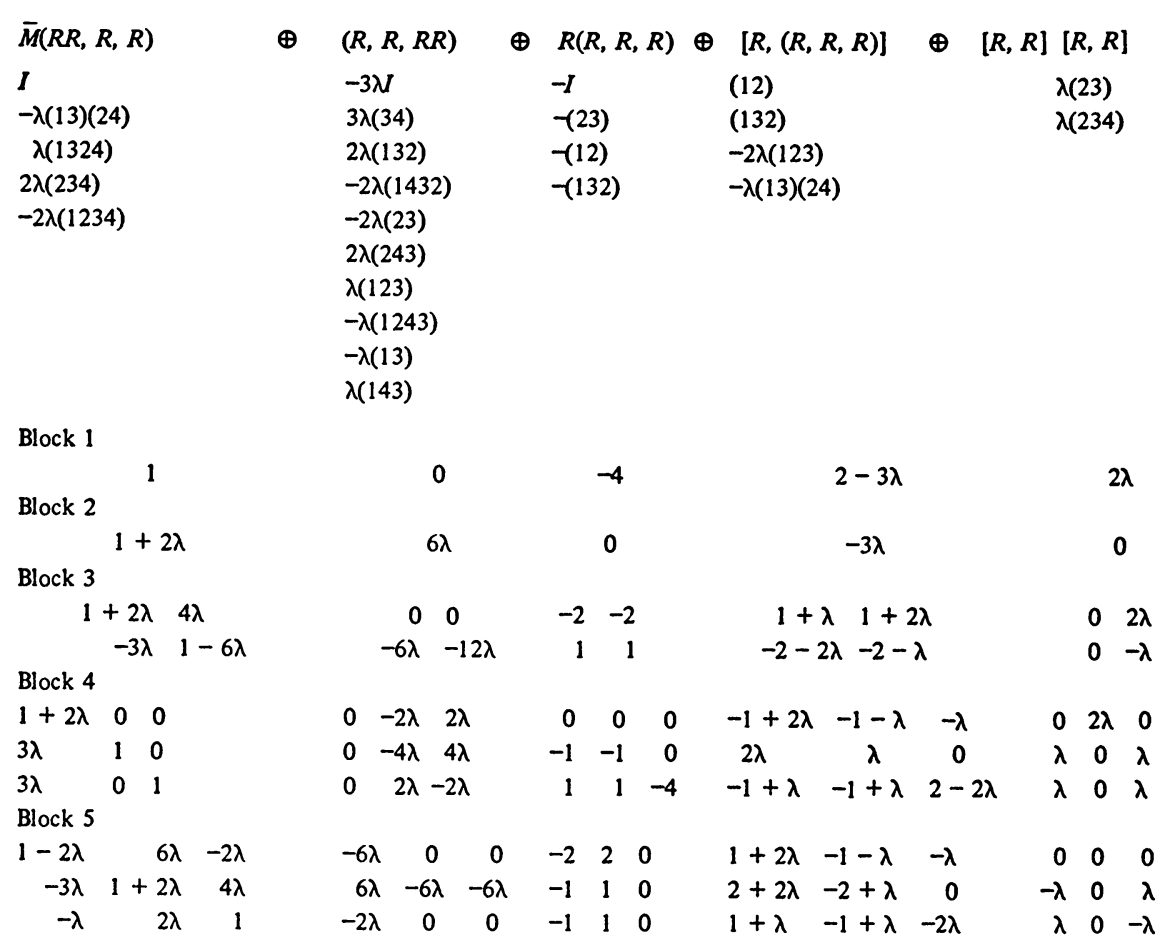


From equation (12) we have that

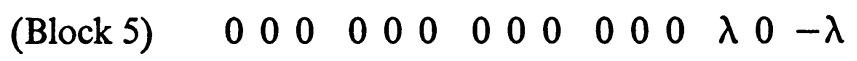

is an identity. Since $\lambda \neq 0,101$ is an identity of Block 5 based on the function $[R, R][R, R]$.

LEMMA 5. If $R$ is a right alternative algebra satisfying equation (1) for $\lambda=-\frac{1}{2}$, then $[a, b][c, d]=[c, d][a, b]$.

Proof. Given a right alternative algebra $(R,+, \cdot)$, define $(R,+, \circ)=$ $R^{+}$by defining $x \circ y=x \cdot y+y \cdot x$. The assumption that $R^{+}$be associative is equivalent to equation (1) with $\lambda=-\frac{1}{2}$. These rings were studied by Thedy in [5, Theorem 7]. He showed that if the algebra were simple, it would be either nil or a field. We shall use the representation of $S_{3}$ given in [2, Table I] based on the functions $(R R) R \oplus R(R R)$.

(i) Right Alternative Law $I+(23) \oplus-I-$ (23),

(ii) equation (1) with $\lambda=-\frac{1}{2}$

$$
\begin{gathered}
I+(12)-\frac{1}{2}(23)-\frac{1}{2}(132)+\frac{1}{2}(123)+\frac{1}{2}(13) \\
\oplus-\frac{1}{2} I-\frac{1}{2}(12)-\frac{1}{2}(23)-\frac{1}{2}(132),
\end{gathered}
$$

(iii) $(a \circ b) \circ c-a \circ(b \circ c)$

$$
I+(12)-(132)-(13) \oplus(123)+(13)-I-(23)
$$

(iv) $(a, b, c)+\frac{1}{2}[a,[b, c]]$

$$
I-\frac{1}{2}(132)+\frac{1}{2}(13) \oplus-\frac{1}{2} I-\frac{1}{2}(23) \text {. }
$$

\begin{tabular}{|c|c|c|}
\hline & $(R R) R$ & $R(R R)$ \\
\hline Block 1 & 1 & -1 \\
\hline Block 2 & 0 & 0 \\
\hline Block 3 & 10 & $\begin{array}{ll}-1 & -1\end{array}$ \\
\hline Diock 3 & $\begin{array}{ll}0 & 1\end{array}$ & 0 \\
\hline
\end{tabular}

One can verify the following equivalences.

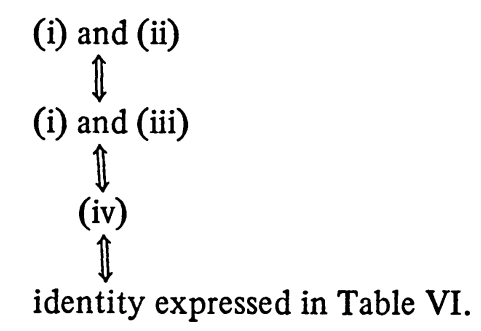

\section{TABLE VI}


We shall need the following identity which is given in [5, equation (4)].

$$
\begin{aligned}
0 \equiv \bar{E}(a, b, c, d)= & (a b, c, d)+(a, b,[c, d]) \\
& -a(b, c, d)-(a, c, d) b .
\end{aligned}
$$

Then

$$
\begin{aligned}
0= & \bar{E}(a, b, c, d)+\frac{1}{2} \bar{C}(a, b,[c, d]) \\
= & \left\{(a b, c, d)+\frac{1}{2}[a b,[c, d]]\right\}-a\left\{(b, c, d)+\frac{1}{2}[b,[c, d]]\right\} \\
& -\left\{(a, c, d)+\frac{1}{2}[a,[c, d]]\right\} b-\frac{1}{2}([c, d], a, b)=-\frac{1}{2}([c, d], a, b)
\end{aligned}
$$

using equation (13). We have established that in a right alternative algebra satisfying equation (1) with $\lambda=-\frac{1}{2}$ then

$$
([R, R], R, R)=0 .
$$

By equation (13) and equation (15) we have $0=([a, b], c, d)=-\frac{1}{2}[[a, b]$, $[c, d]]$. This completes the proof of Lemma 5.

LEMMA 6. If $R$ is a right alternative algebra satisfying equation (1), then $M$ is an ideal and $M^{2}=0$.

Proof. If $\lambda=0$, then $M=0$ and satisfies the condition. Suppose $\lambda \neq 0$. Equation (1) says that $M \subseteq[R,[R, R]]$ and hence $[R, M] \subseteq[R,[R,[R, R]]]$. $\vec{M}([a, b], c, d)-\bar{D}([a, b], c, d)=-\lambda[c,[d,[a, b]]]$ using Lemmas 4 and 5. Thus

$$
[R,[R,[R, R]]] \subseteq M .
$$

By Corollary 1, part (2), $M$ is an ideal.

By equation (1), $M \subseteq[R, R]$. Lemmas 4 and 5 and the fact that $M$ is a two-sided ideal give $0=[M,[R, R]]=[R M,[R, R]]=[M R,[R, R]]$. Let $i$ $\in M, a \in R$, and $c \in[R, R]$. Then $0=\bar{C}(a, i, c)+\bar{C}(i, a, c)+2 \bar{D}(a, i, c)$ $-\bar{A}(a, c, i)-\bar{A}(i, c, a)+\bar{A}(c, a, i)=-2[a, c] i$.

We conclude $[R,[R, R]] M=0$ and, by equation (1), $M^{2}=0$.

Final results. We have established that any semiprime right alternative algebra satisfying equation (1) is an alternative algebra. In this section we will show that when $\lambda \neq 0$ such algebras are associative and commutative.

If $R$ is a right alternative algebra satisfying equation (1) with $\lambda \neq 0$, then both $(R, R, R)$ and $[R,[R, R]]$ are alternating functions of their three arguments $\bmod M$. In any nonassociative ring the following identity holds.

$$
\begin{aligned}
(a, b, c)+ & (b, c, a)+(c, a, b)-(a, c, b)-(c, b, a)-(b, a, c) \\
= & -[a,[b, c]]-[b,[c, a]]-[c,[a, b]] .
\end{aligned}
$$

By the alternating property of both sides $\bmod M$, we deduce $6(a, b, c) \equiv$ 
$-3[a,[b, c]] \bmod M$. From equation (13) we conclude that $R / M$ satisfies equation (1) for $\lambda=-\frac{1}{2}$. We record this as part (a) of the following lemma.

LEMMA 7. Let $R$ be a right alternative algebra satisfying equation (1).

(a) If $\lambda \neq 0, R / M$ satisfies equation (1) for $\lambda=-\frac{1}{2}$.

(b) If $\lambda=-\frac{1}{2}$, then $\langle(R, R, R)\rangle^{2} \subseteq M$.

ProOF OF PART (b). By $\langle(R, R, R)\rangle$ we mean the ideal of $R$ generated by all associators. The nucleus of a nonassociative ring $A$ is the set of all elements $n \in A$ such that $(n, A, A)=(A, n, A)=(A, A, n)=0$. The center of $A$ is the set of all $n \in$ nucleus of $A$ such that $[n, A]=0$. By equation (13) and equation (15) we have $(R, R, R) \subseteq$ nucleus of $R / M$. By equation (13) $[R,(R, R, R)] \subseteq[R,[R,[R, R]]] \subseteq M$ by equation (16). We have shown that $(R, R, R) \subseteq$ center of $R / M$. By $\bar{E}(a, a, b, c)$, we get that $a(a, b, c) \in$ center of $R / M$. Thus $(x, y, a)(a, b, c) \equiv(x, y, a(a, b, c)) \equiv 0 \bmod M$. The function $(R, R, R)(R, R, R)$ is an alternating function of its 6 arguments $\bmod M$. Performing three transpositions gives $(a, b, c)(d, e, f) \equiv-(d, e, f)(a, b, c)$. Since associators are in the center of $R / M$, we have shown $(R, R, R)(R, R, R) \subseteq M$. Again, since the associators are in the center of $R / M,\langle(R, R, R)\rangle^{2} \subseteq M$. This completes the proof of Lemma 7 .

Suppose that $R$ is an associative ring satisfying equation (1) for $\lambda \neq 0$. It is immediate that $[R, R] \subseteq$ center of $R$, and by $\bar{C}(a, a, b)$,

$$
a[a, b] \in \text { center of } R \text {. }
$$

Then $\langle[a, b]\rangle^{2}=\left\langle[a, b]^{2}\right\rangle \subseteq\langle[a[a, b], b]\rangle=0$. We conclude that if $R$ is semiprime, then $R$ is associative and commutative. When $R$ is semiprime, $R$ has no nonzero nilpotent elements, so by [4, Theorem 31, p. 123] $R$ is a subdirect sum of rings without divisors of zero.

THEOREM 2. Let $R$ be a semiprime right alternative algebra satisfying equation (1) for some $\lambda \neq 0$. Then $R$ is associative and commutative. Furthermore, $R$ is a subdirect sum of associative-commutative algebras without divisors of zero.

Proof. Use Lemmas 6 and 7 and the above discussion.

Suppose $R$ is an associative algebra satisfying equation (1) for some $\lambda \neq 0$. We have shown that the ideal generated by any single commutator is an ideal whose square is zero. If $R$ is generated as an algebra by $n$ elements, then $\langle[R, R]\rangle^{[n / 2]+1}=0$ where $[n / 2]$ is the greatest integer in $n / 2$. We will show in general that $\langle[R, R]\rangle$ is not nilpotent.

EXAMPLE. Let $R$ be the free associative algebra over a field $F$ generated by $a_{i}, i=1,2,3, \ldots$ Let $I$ be the ideal generated by $[R,[R, R]]$. Then $\langle[R, R]\rangle$ is not nilpotent $\bmod I$. 
Proof. We shall define a function $\sigma$ on the basis of monomials of $R$ and extend it to all of $R$ by linearity. Every monomial is a product of generators. If the generators in a monomial $w$ are not all distinct, define $\sigma(w)=0$. If the generators of $w$ are all distinct, then $w=\left(a_{i_{1}} a_{i_{2}} a_{i_{3}} \cdots a_{i_{r}}\right) \pi$, where $i_{1} \cdot<i_{2}<$ $i_{3}<\cdots<i_{r}$ and $\pi \in S_{r}$. Define $\sigma(w)=\operatorname{sgn} \pi$. If $w$ is a monomial, we let $|w|$ be the total degree of $w$ in the generators. The sgn function is a degree one representation of $S_{n}$ for any $n$. If it is extended by linearity to $G_{n}$, the group ring of $S_{n}$, it is a ring homomorphism. Notice that if $x \in R$ is a sum of monomials composed of the same $n$ distinct generators, then $x$ may be written as $x=\left(a_{i_{1}} a_{i_{2}} a_{i_{3}} \cdots a_{i_{n}}\right) g$ where $i_{1}<i_{2}<i_{3}<\cdots<i_{n}$ are the indices of the generators, and $g \in G_{n}$. In this case, $\sigma(x)=\operatorname{sgn}(g)$.

We will show that every element of $I$ has $\sigma$ value zero. Every element of $I$ is a sum of terms of the form $w\left[w^{\prime},\left[w^{\prime \prime}, w^{\prime \prime \prime}\right]\right] w^{\prime \prime \prime \prime}$ where each of the $w$ 's is a monomial. If the generators appearing are not all distinct, the $\sigma$ value of the expression is zero. If the generators appearing are distinct, the expression may be written as

$$
\left(a_{i_{1}} a_{i_{2}} a_{i_{3}} \cdots a_{i_{n}}\right) \pi\left\{I-c_{1}^{d_{1}}\right\}\left\{I-c_{2}^{d_{2}}\right\}
$$

$\pi \in S_{n} ; n=\left|w w^{\prime} w^{\prime \prime} w^{\prime \prime \prime} w^{\prime \prime \prime \prime}\right| ; I$ is the identity permutation. $c_{1}$ is a cycle of length $\left|w^{\prime \prime} w^{\prime \prime \prime}\right| . c_{2}$ is a cycle of length $\left|w^{\prime} w^{\prime \prime} w^{\prime \prime \prime}\right| . d_{1}=\left|w^{\prime \prime \prime}\right| . d_{2}=\left|w^{\prime \prime} w^{\prime \prime \prime}\right|$. If $\left|w^{\prime \prime} w^{\prime \prime \prime}\right|$ is odd, then $c_{1}$ is an even permutation and $\operatorname{sgn}\left(I-c_{1}^{d_{1}}\right)=0$; if $\left|w^{\prime \prime} w^{\prime \prime \prime}\right|$ is even, then $c_{2}^{d_{2}}$ is an even permutation, so $\operatorname{sgn}\left(I-c_{2}^{d_{2}}\right)=0$. In any case, $\sigma\left(w\left[w^{\prime},\left[w^{\prime \prime}, w^{\prime \prime \prime}\right]\right] w^{\prime \prime \prime \prime}\right)=0$.

We have shown that $\sigma(I)=0$.

$$
\sigma\left(\left[a_{1}, a_{2}\right]\left[a_{3}, a_{4}\right]\left[a_{5}, a_{6}\right] \cdots\left[a_{2 n+1}, a_{2 n+2}\right]\right)=2^{n+1} \neq 0 .
$$

Thus $[R, R]^{n} \subseteq I$ for any $n$, and hence $\langle[R, R]\rangle$ is not a nilpotent ideal.

The author wishes to thank the referee for the helpful suggestions. With these suggestions, the author was able to include the exceptional cases of $\lambda=-\frac{1}{2}$ and $\lambda=-\frac{3}{8}$.

The author also wishes to thank his wife for her skillful proofreading and editing.

\section{REFERENCES}

1. L. Dornhoff, Group representation theory, Pure and Applied Mathematics, Marcel Dekker, New York, 1971.

2. I. Hentzel, Processing identities by group representation, Special Session on Computers in Nonassociative Rings and Algebras, Amer. Math. Soc. 82nd Annual Meeting, San Antonio, Texas, 1976.

3. I. Hentzel, G. Piacentini Cattaneo and D. Floyd, Alternator and associator ideal algebras, Trans. Amer. Math. Soc. 229 (1977), 87-109.

4. N. McCoy, Rings and ideals, The Carus Mathematical Monographs, No. 8, Math. Assoc. of America.

5. A. Thedy, Right alternative rings, J. Algebra 37 (1975), 1-43.

Department of Mathematics, Iowa State University, Ames, Iowa 50011 